\title{
White dwarfs in the Gaia era
}

\author{
P.-E. Tremblay ${ }^{1}$, N. Gentile-Fusillo ${ }^{1}$, J. Cummings ${ }^{2}$, S. Jordan ${ }^{3}$, \\ B. T. Gänsicke ${ }^{1}$ and J. S. Kalirai ${ }^{2,4}$ \\ ${ }^{1}$ Department of Physics, University of Warwick, \\ CV4 7AL, Coventry, UK \\ email: P-E.Tremblay@warwick.ac.uk \\ ${ }^{2}$ Center for Astrophysical Sciences, Johns Hopkins University, \\ 3400 North Charles Street, Baltimore, MD 21218, USA \\ ${ }^{3}$ Astronomisches Rechen-Institut, Zentrum für Astronomie der Universität Heidelberg, \\ D-69120 Heidelberg, Germany \\ ${ }^{4}$ Space Telescope Science Institute, \\ 3700 San Martin Drive, Baltimore, MD 21218, USA
}

\begin{abstract}
The vast majority of stars will become white dwarfs at the end of the stellar life cycle. These remnants are precise cosmic clocks owing to their well constrained cooling rates. Gaia Data Release 2 is expected to discover hundreds of thousands of white dwarfs, which can then be observed spectroscopically with WEAVE and 4MOST. By employing spectroscopically derived atmospheric parameters combined with Gaia parallaxes, white dwarfs can constrain the stellar formation history in the early developing phases of the Milky Way, the initial mass function in the 1.5 to $8 M_{\odot}$ range, and the stellar mass loss as well as the state of planetary systems during the post main-sequence evolution.
\end{abstract}

Keywords. astrometry, white dwarfs, solar neighborhood, Galaxy: stellar content, Galaxy: evolution

\section{Background}

The European Space Agency (ESA) astrometric mission Gaia will determine positions, parallaxes, and proper motions for a full sky sample with $V \lesssim 20$ mag, corresponding to $\sim 1 \%$ of the stars in the Galaxy (Gaia Collaboration et al. 2016a). In addition to astrometric measurements, the Gaia catalog will include $G$ passband photometry, lowresolution spectrophotometry in the blue (BP, 330-680 nm) and red (RP, 640-1000 nm), and (for bright stars, $G \lesssim 15$ ) higher-resolution spectroscopy in the region around $860 \mathrm{~nm}$ with the Radial Velocity Spectrometer (Jordi et al. 2010, Carrasco et al. 2014). The final data release is expected to include between 250,000 and 500,000 white dwarfs (Torres et al. 2005, Carrasco et al. 2014).

Gaia DR1 was limited to $G$ passband photometry and the astrometric solution for stars in common with the Hipparcos and Tycho-2 catalogs (Gaia Collaboration et al. 2016b). However, not all Hipparcos and Tycho-2 stars are found in Gaia DR1 owing to source filtering. Tremblay et al. (2017) recovered only 6 directly observed white dwarfs but they have also selected 46 members of wide binaries for which the companion has a precise Gaia DR1 parallax. They compared observed and predicted spectroscopic distances and found that the mean agreement is better than $1 \%$ between Gaia parallaxes, published atmospheric parameters, and theoretical mass-radius relations (Fontaine et al. 2001). In this work, we examine the prospects of studying degenerate stars in Gaia DR2 planned for April 2018. 

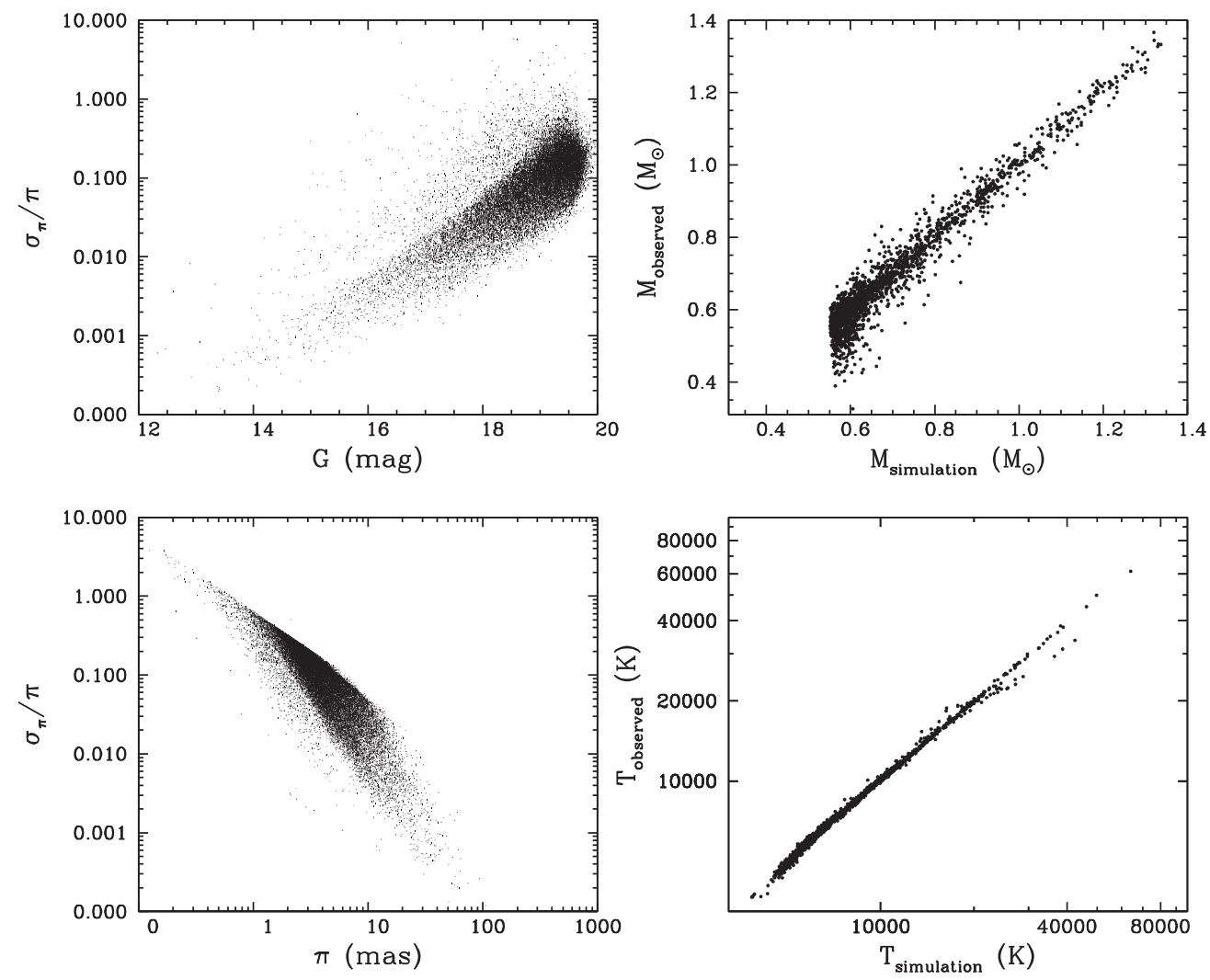

Figure 1. (Left:) Gaia DR2 parallax errors as a function of $G$-band magnitude (top left) and parallax (bottom left) for our simulated Galactic white dwarf population. (Right:) Photometric fit for all white dwarfs within $100 \mathrm{pc}$ in our Gaia DR2 mock catalog. We rely on Gaia data alone and our simulation does not include binary evolution. Gaia colours are predicted assuming a ratio of $1 / 4$ between $\mathrm{He}-$ and $\mathrm{H}$-atmospheres.

\section{White dwarfs in Gaia Data Release 2}

Gaia DR2 is expected to have the astrometric solutions and integrated $G, G_{\mathrm{BP}}$ and $G_{\mathrm{RP}}$ photometric fluxes for all sources with acceptable formal standard errors. It will have significantly higher individual precision due to a longer measurement time (22 months instead of 11 months, which is already $36 \%$ of the total mission time). Systematic errors are also expected to decrease significantly resulting from a more sophisticated calibration, including a better definition of the line spread function, the application of a chromaticity correction, a more accurate calibration of the basic angle variation, and a calibration and correction of micro clanks.

We have simulated the Gaia DR2 white dwarf population using assumptions similar to those presented in Tremblay et al. (2016) for the magnitude-limited SDSS sample. The main difference is that Gaia is all-sky and it is currently expected that remnants of all colours will be released in DR2 given the discussion above. Our simulation is similar to those presented in Torres et al. (2005) and Carrasco et al. (2014), except that we use the most recent predicted DR2 errors on parallaxes and colours drawn from PyGaia†. The total number of white dwarfs in Gaia DR2 is expected to be between 250,000 and 500,000 depending on the Galactic model. In particular, by extrapolating the current 


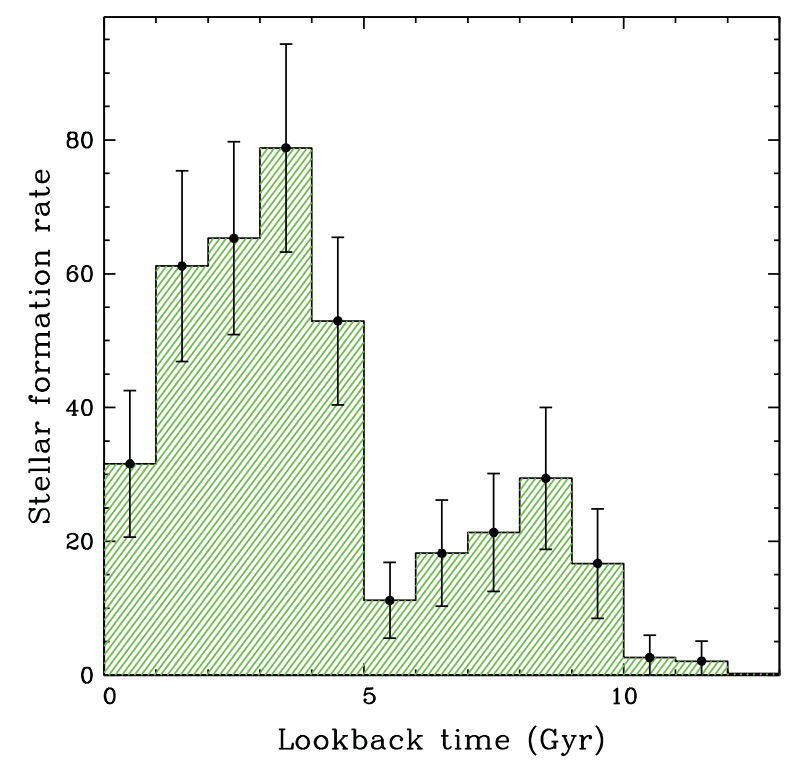

Figure 2. Derived total stellar formation rate (relative number) as a function of lookback time for the Galactic disk at $8 \mathrm{kpc}$ from Galactic centre (see Tremblay et al. 2014). The source of the data is the volume complete $20 \mathrm{pc}$ white dwarf sample (Giammichele et al. 2012) and the initial-to-final mass relation of Kalirai et al. $(2008,2009)$ to convert white dwarf parameters to initial stellar parameters. We accounted for biases due to the missing main-sequence stars and the velocity dispersion as a function of total stellar age in the direction perpendicular to the plane of the disk. The error bars take into account number statistics uncertainties and are derived from the uncorrected number of white dwarfs. We do not account for radial mixing and as such this represents the formation of stars that are at present day at $8 \mathrm{kpc}$ Galactic radius.

remnant space density in the complete 13 pc sample (Holberg et al. 2016), we obtain $\sim 2200$ white dwarfs within $50 \mathrm{pc}$, the maximum distance for which we can hope for a volume complete DR2 sample (Carrasco et al. 2014).

Fig. 1 (left panel) illustrates the Gaia DR2 parallax precision as a function of $G$-band magnitude and parallax for our mock white dwarf catalog. It is noted in particular that all white dwarfs within $100 \mathrm{pc}$ will have a distance precision better than $\sim 4 \%$. We performed Gaia data only photometric fits of all simulated remnants within $100 \mathrm{pc}$. Gaia colours were predicted assuming a ratio of $1 / 4$ between He- and H-atmospheres. Fig. 1 (right panel) demonstrates that it is possible to extract white dwarf parameters within a few percent from Gaia data alone within 100 pc. It is observed that Gaia integrated photometric fluxes are not very sensitive to the atmospheric composition since the bandpasses are broad, yet the photometric precision is large enough for accurate $T_{\text {eff }}$ determinations. We note that our mock catalog does not include binary evolution, and as such the actual Gaia catalog is expected to have a significant number of outliers when using single white dwarf models.

\section{Future outlook}

Starting with DR2 it will be feasible to compare predicted spectroscopic distances (Holberg et al. 2008) for large samples of pure-hydrogen (DA) and pure-helium atmosphere remnants (DB) with Gaia parallaxes. This will effectively test white dwarf atmosphere and structure models at the $1 \%$ level over a wide range of cooling ages and masses. It will also be possible to derive precise mass distributions for other white dwarf subtypes, 
such as cool metal rich objects (DZ, DQ) and magnetic remnants, providing crucial information on the internal structure and progenitors of these spectral types. Furthermore, Gaia DR2 will be invaluable in characterising the binary white dwarf population (Toonen et al. 2017) and identifying a halo white dwarf sample from proper motions and calculate its luminosity function (Cojocaru et al. 2015). Most of these applications can only be performed for a small part of the Gaia DR2 sample with currently available independent spectroscopic observations. WEAVE (Dalton et al. 2016) and 4MOST (de Jong et al. 2014) spectroscopic follow-up surveys will thus have a major impact in enlarging the science output from Gaia white dwarfs.

Gaia DR2 will make it possible to update the local stellar formation history shown in Fig. 2 as derived from white dwarfs within 20 pc (Tremblay et al. 2014). The Gaia sample within 100 pc will be 10 times larger, significantly reducing the error bars from number statistics. Once again, WEAVE and $4 M O S T$ spectroscopic follow-ups will be essential to improve the precision of the conversion of white dwarf parameters to initial stellar parameters from the initial-to-final mass relation (Cummings et al. 2016). Gaia will evidently allow for a strong synergy between white dwarf research and Galactic archeology.

\section{Acknowledgement}

This work has made use of data from the European Space Agency (ESA) mission Gaia (https://www.cosmos.esa.int/gaia), processed by the Gaia Data Processing and Analysis Consortium (DPAC, https://www.cosmos.esa.int/web/gaia/dpac/consortium). Funding for the DPAC has been provided by national institutions, in particular the institutions participating in the Gaia Multilateral Agreement.

\section{References}

Bergeron, P., Saffer, R. A., \& Liebert, J. 1992, ApJ, 394, 228

Carrasco, J. M., Catalán, S., Jordi, C., et al. 2014, A\&A, 565, A11

Cojocaru, R., Torres, S., Althaus, L. G., Isern, J., \& García-Berro, E. 2015, A\& A, 581, A108

Cummings, J. D., Kalirai, J. S., Tremblay, P.-E., Ramirez-Ruiz, E., \& Bergeron, P. 2016, ApJL, 820, L18

Dalton, G., Trager, S., Abrams, D. C., et al. 2016, Proceedings of the SPIE, 9908, 99081G

Fontaine, G., Brassard, P., \& Bergeron, P. 2001, PASP, 113, 409

Gaia Collaboration, Brown, A. G. A., Vallenari, A., et al. 2016, A\&AA, 595, A2

Gaia Collaboration, Prusti, T., de Bruijne, J. H. J., et al. 2016, A\&A, 595, A1

Giammichele, N., Bergeron, P., \& Dufour, P. 2012, ApJS, 199, 29

Holberg, J. B., Bergeron, P., \& Gianninas, A. 2008, AJ, 135, 1239

Holberg, J. B., Oswalt, T. D., Sion, E. M., \& McCook, G. P. 2016, MNRAS, 462, 2295

de Jong, R. S., Barden, S., Bellido-Tirado, O., et al. 2014, Proceedings of the SPIE, 9147, 91470M

Jordi, C., Gebran, M., Carrasco, J. M., et al. 2010, A\&A, 523, A48

Kalirai, J. S., Hansen, B. M. S., Kelson, D. D., et al. 2008, ApJ, 676, 594

Kalirai, J. S., Saul Davis, D., Richer, H. B., et al. 2009, ApJ, 705, 408

Toonen, S., Hollands, M., Gaensicke, B. T., \& Boekholt, T. 2017, A\&A, in press, arXiv: 1703.06893

Torres, S., García-Berro, E., Isern, J., \& Figueras, F. 2005, MNRAS, 360, 1381

Tremblay, P.-E., Kalirai, J. S., Soderblom, D. R., Cignoni, M., \& Cummings, J. 2014, ApJ, 791, 92

Tremblay, P.-E., Cummings, J., Kalirai, J. S., et al. 2016, MNRAS, 461, 2100

Tremblay, P.-E., Gentile-Fusillo, N., Raddi, R., et al. 2017, MNRAS, 465, 2849 